\title{
ABSTRACTS OF CONTRIBUTED PAPERS
}

THE UNIFORMITY OF CHEMICAL COMPOSITION OF GALACTIC PLANETARY NEBULAE

\author{
Timothy Barker \\ Wheaton College
}

The relative abundances of $\mathrm{H}$, $\mathrm{He}, \mathrm{Ne}, \mathrm{O}$, and $\mathrm{N}$ have been determined in thirty planetary nebulae of widely-differing kinematical properties. Helium abundances are found to be affected by incomplete ionization of $\mathrm{He}$, an effect that is strongly correlated with the abundance of $\mathrm{S}^{+}$. The He and $\mathrm{N}$ abundances appear to be positivelycorrelated, suggesting that nebular material has been contaminated through mixing with CNO-processed material. There is some evidence for a radial galactic abundance gradient in $\mathrm{He}, \mathrm{N}$, and possibly $\mathrm{O}$ and $\mathrm{Ne}$; abundances increase toward the gallactic center. This gradient is evident only from the nebulae that appear to be moving in circular orbits. A11 nebulae (except the previously studied planetary in the galactic halo and the one in the globular cluster M 15) have nearly the same abundances of 0 and $\mathrm{Ne}$ as galactic HII regions and the sun, and there is little correlation between these abundances and the kinematical properties of the planetaries. Apparently either $\mathrm{Ne}$ and 0 are not representative of the true heavy metal abundance, or the vast majority of planetaries belong to a metal-rich population. The latter possibility seems more likely in view of recent observations of the $\mathrm{S}^{++}$abundances of nineteen of the nebulae, which show them to have generally solar $S$ abundances. (Paper will appear in The Astrophysical Journal.)

IMPROVED ABUNDANCES IN THREE HALO PLANETARY NEBULAE

Steven A. Hawley, Joseph S. Miller

Lick Observatory, University of California, Santa Cruz

With the Lick Observatory $3 \mathrm{~m}$ telescope and image dissector scanner we have obtained new spectrophotometric observations of three halo planetary nebulae; $49+88^{\circ} 1,108-76^{\circ} 1$, and $K 648$. Based on the observed line intensities we are able to compute temperatures, densities, and abundances of $\mathrm{He}, \mathrm{O}, \mathrm{N}$, and $\mathrm{Ne}$. Our data show that the helium abundance in the halo planetaries is normal with respect to disk planetaries. Oxygen is consistently less abundant in the halo planetaries by factors of 4,12 , and 20 . The ratio $N / 0$ in $49+88^{\circ} 1$ and $K 648$ is 